$16^{\text {th }}$ International Conference on

AEROSPACE SCIENCES \& AVIATION TECHNOLOGY,

ASAT - 16 - May 26 - 28, 2015, E-Mail: asat@ mtc.edu.eg Military Technical College, Kobry Elkobbah, Cairo, Egypt

Tel : +(202) 24025292 - 24036138, Fax: +(202) 22621908

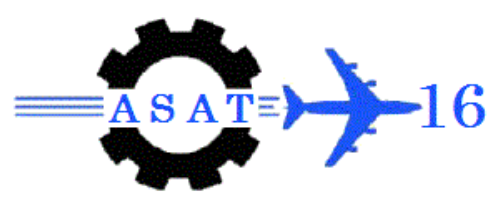

\title{
Finite Element Computations of Complex Stress Intensity Factor Magnitude of Interfacial Crack in Bi-Material Composites
}

\author{
S. A. K. Yossif*
}

\begin{abstract}
Composite structures are distinguished by interfaces separating their different layers. These interfaces represent region of mismatches in mechanical and thermal properties of the adjacent layers. These regions are rich in cracks which are laying either on or sub- the interface in a direction parallel or perpendicular to it. In bi-material composites, interfacial crack is under mixed loading mode and stress intensity factor is a complex number even if the opening mode loading is applied. This paper solves for energy release rates and the magnitude of complex intensity factor of interfacial cracks using the classical stiffness finite element method. The path-independent $J$ integral is used to calculate the energy release rate during crack extension in the elastic plane strain deformation field. In this work, the crack tip region is meshed with fan-shaped non-singular four nodes isoparametric quadrilateral elements. In addition, a conical shape of $q$ function is employed in the numerical implementation of the equivalent energy domain integral. Realization of all numerical computations and demonstration of results are completely composed and written in MATLAB language. Meshing the computational domains and crack tip region are performed by a free downloadable program AUTOMESH-2D. Numerical results of stress intensity factor are found to be very close to the analytical and referenced values in both cases of bi-material and single layer systems. Furthermore, numerical values of $J$-integral contours are very close to interfacial fracture energy measured experimentally between a hard film and a soft substrate.
\end{abstract}

Keywords: Bi-material composite, interfacial crack, $J$ integral, complex stress intensity factor, fracture mechanics, finite element method.

\section{INTRODUCTION}

Owing to its importance in practical applications, such as thermal barrier coatings, layered structures in aerospace and naval industry, welding of two dissimilar solids ...etc., solution of interfacial crack problems between two dissimilar elastic materials has attracted interests of many scientists and researchers, $[1,2,3]$. They proved that the stress fields near the tip of interfacial crack are singular, depend on mechanical properties of both boned layers and have an oscillatory nature. The singular stresses field ahead the interfacial crack tip; i.e. $\theta=0$; under remote normal $\sigma_{y}^{\infty}$ and shear $\tau_{x y}^{\infty}$ stresses, Fig. 1, are calculated as the following, [4]:

$\sigma_{y}+i \tau_{x y}=\frac{K}{\sqrt{2 \pi r}} r^{i \epsilon}$

\footnotetext{
*Lecturer at Mechanical Design Department, Faculty of Engineering - Mataria - Helwan University - Cairo - Egypt. Email: salah.aleasha@ hotmail.com
} 
Where $i=\sqrt{-1}, K$ is the complex stress intensity factor, and $\epsilon$ is the bi-material (bi-elastic) constant. The bi-material constant or the oscillation index $\epsilon$ represents the mismatch in mechanical properties between the two layers at the interface; it is evaluated according to, [5]:

$\epsilon=\frac{1}{2 \pi} \ln \left[\frac{G_{1}+G_{2} \kappa_{1}}{G_{2}+G_{1} \kappa_{2}}\right]$

Where $G$ is shear modulus, and:

$\kappa=\left\{\begin{array}{cc}\frac{3-v}{1+v} & \text { for plane stress } \\ 3-4 v & \text { for plane strain }\end{array}\right.$

Subscripts 1 and 2 refer to the material above and below the interface respectively. The expression of complex intensity factor is, [6]:

$K=K_{1}+i K_{2}=\left(\sigma_{y}^{\infty}+i \tau_{x y}^{\infty}\right)(1+2 i \epsilon) \sqrt{\pi a}(2 a)^{-i \epsilon}$

Where $K_{1}=\operatorname{Re}(K)$, and $K_{2}=\operatorname{Im}(K)$ are the real and imaginary parts of $K$ respectively.

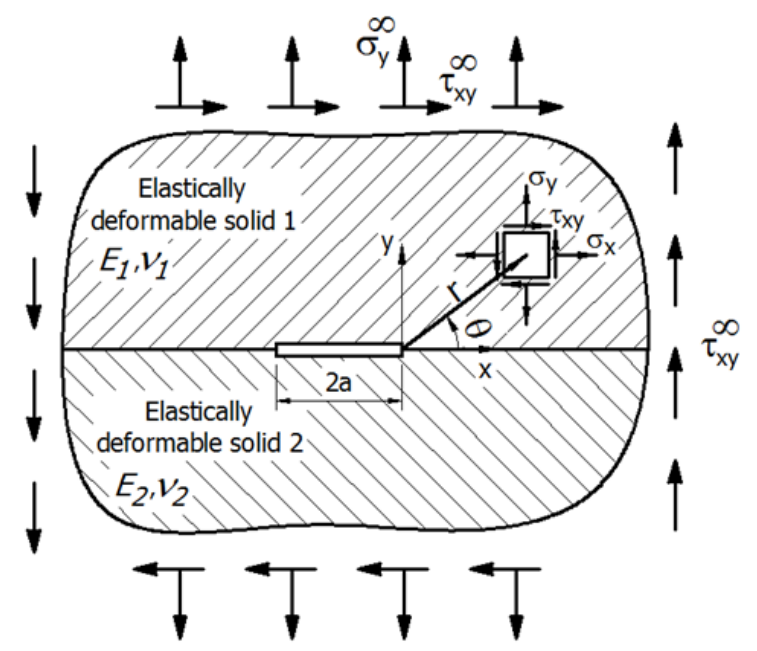

Fig. 1 Interfacial Crack Between Two Dissimilar Perfectly Bonded Elastic Solids.

The magnitude of complex stress intensity factor is:

$\mathcal{K}=|K|=\sqrt{K_{1}^{2}+K_{2}^{2}}$

If the shear stress component in Eq. (3.a) is nullified, i.e. $\tau_{x y}^{\infty}=0$, as in the case of the present study, the expression of complex stress intensity factor becomes:

$K=K_{1}+i K_{2}=\sigma_{y}^{\infty} \sqrt{\pi a}(1+2 i \epsilon)(2 a)^{-i \epsilon}$

Equation (4) states that $K$ is inseparable into the opening and shearing modes even the loading is perpendicular to the interface of the bi-material composite. In other words, in the bimaterial system, due to the mismatch in mechanical properties at the interface; i.e. $\epsilon \neq 0$, the crack mode is mixed. In the case of a single material system, $\epsilon$ is equal to zero and the expression of $K$ in Eq. (3.a), becomes: 


$$
K=K_{1}+i K_{2}=\left(\sigma_{y}^{\infty}+i \tau_{x y}^{\infty}\right) \sqrt{\pi a}=K_{I}+i K_{I I}
$$

In this case, the expressions of $K_{1}$ and $K_{2}$ have the same physical meaning of the familiar stress intensity factors $K_{I}$ and $K_{I I}$ which are corresponding to the opening and shearing modes respectively. The crack face displacement at a distance $r$ behind the crack tip is calculated by the following equation, [7]:

$\delta_{2}+i \delta_{1}=\frac{8}{(1+2 i \epsilon) \cosh \pi \epsilon} \frac{\left(K_{1}+i K_{2}\right)}{E^{*}} \sqrt{\frac{r}{2 \pi}} r^{i \epsilon}$

Where $\delta_{1}=u(r, \pi)-u(r,-\pi)$ and $\delta_{2}=v(r, \pi)-v(r,-\pi) . u$ and $v$ are displacement components along $x$ and $y$ directions respectively. The term $E^{*}$ is defined as the mean Young's modulus of the bi-material composite, and is evaluated according to, [7]:

$E^{*}=\frac{2 \bar{E}_{1} \bar{E}_{2}}{\bar{E}_{1}+\bar{E}_{2}}$

Where:

$\bar{E}=\left\{\begin{array}{cc}E & \text { for plane stress } \\ \frac{E}{1-v^{2}} & \text { for plane strain }\end{array}\right.$

Inspection of equations (1) and (6) reveals that both stresses and deformations fields are synchronously oscillating at the interface, where the term $r^{i \varepsilon}$ may be decomposed into two harmonic waves; i.e. $r^{i \varepsilon}=\cos (\varepsilon \ln r)+i \sin (\varepsilon \ln r)$. As a sequence, equation (6) implies interpenetration of one layer into another in the vicinity of the crack tip. However, for most bi-material systems, the values of $\epsilon$ are multiplications of 0.01 , for example for $\mathrm{Al}_{2} \mathrm{O}_{3} / \mathrm{Cu}$ system $\epsilon=-0.0283$, and $\epsilon=-0.039$ is for $\mathrm{Al}_{2} \mathrm{O}_{3} / \mathrm{Ti}$ system, [8]. These low values of $\epsilon$ attenuate the frequency of stress oscillations and the consequent interpenetrations. Thus, both phenomena are neglected in the present study. The energy release rate for the elastic crack advance at the interface is, [7]:

$\mathbb{G}=\frac{1}{\cosh ^{2}(\pi \epsilon)} \frac{\left(K_{1}^{2}+K_{2}^{2}\right)}{E^{*}}$

Equation (8) is reduced to $\mathbb{G}=\left(K_{I}^{2}+K_{I I}^{2}\right) / E^{*}$ for homogenous solid, i.e. when $\epsilon=0$. The magnitude of stress intensity factor, equation (3.b), can be calculated from the energy release rate; equation (8) as the following:

$\mathcal{K}=\cosh (\pi \epsilon) \sqrt{E^{*} \mathbb{G}}$

In modern structures, where materials, geometries, interfaces, loading and boundary conditions are heterogeneous and nonlinear, application of the aforementioned analytical solutions might be difficult. Consequently, numerical solutions are recommended. The assumed - displacement or stiffness finite element method seizes a prestigious position in solving many problems related to solid and structural mechanics and it could be efficient candidate in the field of fracture mechanics as well. However, some recommendations for meshing the cracked regions to simulate singular stress fields around the crack tip are required. Besides, advanced methodologies for calculating crack characteristics are supplemented. In two-dimensional problems, it is customary to mesh the cracked model with 
high order isoparametric quadrilateral elements having at least eight or higher number of nodes. Such element should have at least one node on each element's edge. In order to simulate the $\frac{1}{\sqrt{r}}$ singularity of strain and stress fields around the crack tip, the corner nodes may be collapsed to the crack tip forming a rosette of fan-shaped triangular elements. In addition, the edge nodes are displaced by $\frac{l}{4}$ towards the crack tip where $l$ is the length of the element edge. Such elements are called singular. Details and list of many relevant papers to this approach are extensively explained in references [9] and [10]. Another familiar methodology in solving crack problems is to use the extended finite element method (XFEM). The standard displacement-based approximation is enriched near a crack by incorporating discontinuous and the near tip asymptotic fields through a partition of unity method. Comprehensive information about X-FEM can be found in references [11 - 14].

In this paper, the complex stress intensity factor and energy release rate of through thickness interfacial crack are numerically calculated. The displacement-based or stiffness finite element method is used to calculate deformation, strain and stress fields. Plane strain state of deformation is assumed. Materials of the composite system are linear, isotropic and elastic. The layers are meshed by four nodes isoparametric quadrilateral elements. The four nodes quadrilateral elements are degenerated to three nodes nonsingular triangular elements at the crack tip. Four parameters are introduced to design crack tip mesh. The energy release rate is calculated using the energy domain area integral which is equivalent to the contour path independent $J$-integral. Conical form of $q$ function is proposed to calculate the equivalent energy domain integral. Magnitude of stress intensity factor is calculated through equation (9). In the present work, all numerical computations including realization of the finite element model, proposed $q$ function, equivalent energy domain integral and demonstration of results are completely composed and written in MATLAB R2012a language. Meshing the computational domains and crack tip region are performed by a free downloadable program AUTOMESH-2D [15]. The second section explains briefly the principals of $J$ - integral and the equivalent energy domain area integral. The third section explains briefly shape function of four nodes isoparametric quadrilateral element and derivation of element stiffness matrix and force vector. The fourth section explains the meshing of crack tip region and numerical realization of $q$ function. The fifth section solves three different crack problems and compares the results with referenced values in order to check the validation of the proposed technique.

\section{PATH INDEPENDENT $\boldsymbol{J}$ INTEGRAL METHOD}

Rice [16] proved that the energy release rate of a crack extension in a nonlinear elastic solid is equal to the value of a path independent contour integral performed round that crack. The contour integral is denoted by $J$ - integral and has the following mathematical expression, [16]:

$J=\int_{\Gamma}\left(w d y-\mathbf{T} \cdot \frac{\partial \mathbf{u}}{\partial \mathbf{x}} d s\right)$

Where $w=w(x, y)=w(\varepsilon)=\int_{0}^{\varepsilon} \sigma_{i j} d \varepsilon_{i j}$ is the strain energy density, $\sigma_{i j}$ is the stress tensor, $\varepsilon_{i j}$ is the strain tensor, $\mathbf{u}=(u, v)$ is displacement vector, $\mathbf{x}=\left(x_{1}, x_{2}\right)=(x, y)$ is spatial coordinate system, $\mathbf{T}=\sigma_{i j} n_{j}$ is the traction vector, $n_{j}$ is component of normal vector to closed contour $\Gamma$, and $d s$ is an element of arc length along $\Gamma$, Fig. 2(a). Rice proved that value of $J$-integral is equal to the energy release rate of a crack extension in a deformed nonlinear elastic solid; i.e.:

$\mathbb{G}=J$ 
Rice stated that the value of $J$ integral around a closed contour in nonlinear elastic defect-free solid is zero. Apply Eq. (10) to the crack problem in Fig. 2(b), we get:

$J=J_{\Gamma_{1}}+J_{\Gamma_{2}}+J_{\Gamma_{3}}+J_{\Gamma_{4}}=0$

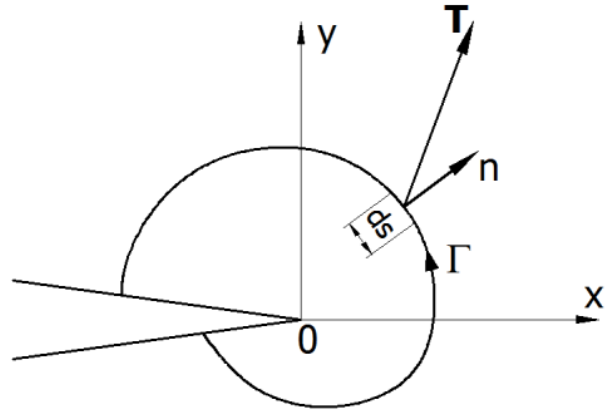

(a)

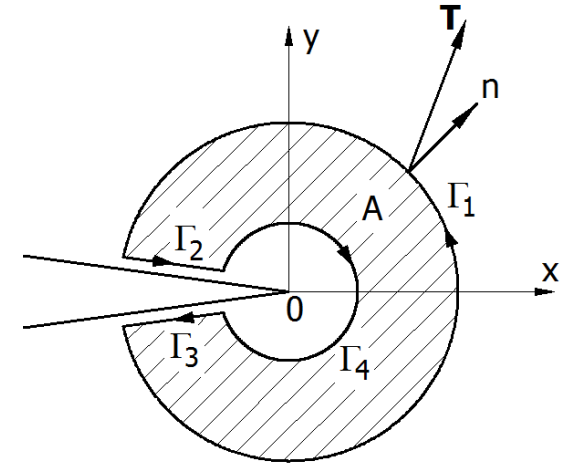

(b)

Fig. 2 (a) Definition of $J$ integral (b) Application of $J$ integral in crack problems.

Since the crack faces are traction free; i.e. $\mathbf{T}_{\Gamma_{2}}=\mathbf{T}_{\Gamma_{3}}=0$, and the change in vertical heights is zeros; i.e. $d y_{\Gamma_{2}}=d y_{\Gamma_{3}}=0$, then we have:

$J_{\Gamma_{2}}=J_{\Gamma_{3}}=0$

Substituting by Eq. (12.b) into (12.a), we get:

$J_{\Gamma_{1}}=-J_{\Gamma_{4}}$

In words, the values of $J$ integral carried along either $\Gamma_{1}$ or $\Gamma_{4}$ are equal. Equation (12.c) proves the path independency of $J$ integral when applied to a crack problem, the negative sign appears because the direction of $\Gamma_{1}$ is opposite to that of $\Gamma_{4}$, Fig. 2(b).

However, the form of $J$ integral, which appears in equation (10), is not suitable for FEM implementation [17]. Divergence theorem of Gauss is used - in two-dimensional space - to transform the domain of the integration from a contour $\Gamma$ onto the area $A$ enclosed by that contour, Fig. 2(b), mathematically, [18]:

$J=\int_{A}\left[\sigma_{i j} \frac{\partial u_{j}}{\partial x_{1}}-w \delta_{1 i}\right] \frac{\partial q}{\partial x_{i}} d A$

Where $\delta_{i j}$ is the Kronecker delta, and $q$ is a mathematical device that enables the generation of an area integral. Equation (13) is called the equivalent energy domain integral. Realization of $q$ function is deeply explained in the fourth section.

\section{REALIZATION BY THE FINITE ELEMENT METHOD}

In this work, the bi-material composite is subjected to a remote vertical tensile stress perpendicular to its interface. A through thickness crack of length $2 a$ exists at the interface. The crack faces are traction free. The strain and stress fields are in the state of plane strain condition. Figure 3.a illustrates the full mathematical model of the interfacial crack problem shown in Fig. 1. Both layers have the same length $2 b$ and width of $h$ The applied surface tractions are $\sigma_{y}^{\infty}=\sigma_{y}$ and $\tau_{x y}^{\infty}=0$. According to the prescribed geometries and applied surface traction, the finite element model would be symmetric, as shown in Fig. 3(b). 
Horizontal displacement of the left edge nodes and the vertical displacement of the bottom edge nodes are fixed. A surface traction $\sigma_{y}$ is applied on the top edge of the model, Fig. 3(b). The crack is simulated as an isosceles triangle cut from the symmetric model as shown in Fig. 3(b). The angle $\varphi$ between crack faces is recognized as a crack mouth angle. The height of the isosceles triangle is equal to the half crack length, Fig. 3(b). A Cartesian coordinate system oxy is fixed at the crack tip, Fig. 3(b) The whole domain is discretized by the four nodes isoparametric quadrilateral elements which is abbreviated by QUAD4. Figure 4 shows the QUAD4 element in the Cartesian coordinate system oxy and its mapped image in natural, intrinsic or normalized coordinate system $o \xi \eta$. Most of forthcoming formulations of QUAD4 are taken from [19]. The spatial coordinates $x$ and $y$ of QUAD4 vary according to:

$x=N_{i}(\xi, \eta) x_{i}$ and $y=N_{i}(\xi, \eta) y_{i}$

Where $x_{i}$ and $y_{i}$ are nodal coordinates in oxy Cartesian coordinate system, Fig. 4(a); and $i=1,2,3,4 . N_{i}(\xi, \eta)$ are the shape functions of the element; defined as:

$N_{i}=\frac{1}{4}\left(1+\xi \xi_{i}\right)\left(1+\eta \eta_{i}\right)$

Where $\xi_{i}$ and $\eta_{i}$ are nodal coordinates in $o \xi \eta$ natural coordinate system, Fig. 4(b).

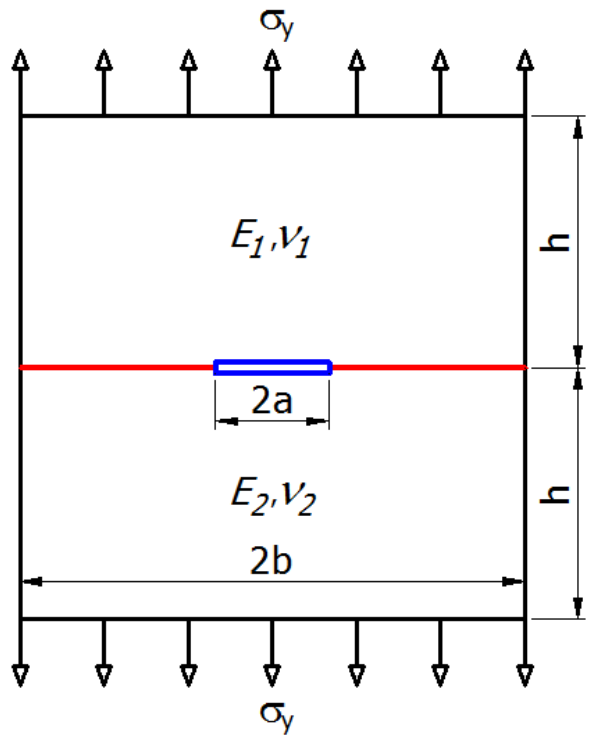

(a)

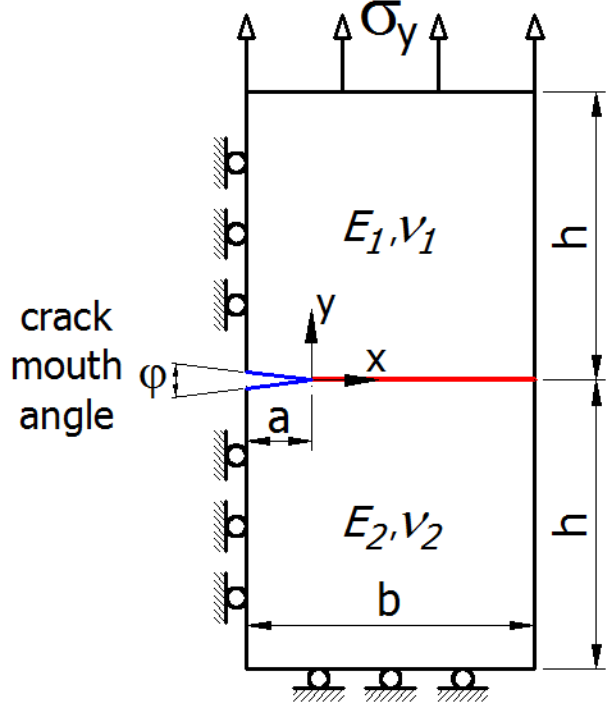

(b)

Fig. 3 (a) Full Mathematical Model. (b) Half Symmetric Model: Applied Surface Traction and Boundary Conditions.

The horizontal $u(x, y)$ and vertical $v(x, y)$ displacement fields within the element are:

$\left\{\begin{array}{l}u \\ v\end{array}\right\}=\mathbf{N d}$

Where $\mathbf{N}=\left[\begin{array}{cc}N_{i} & 0 \\ 0 & N_{i}\end{array}\right]$ is shape matrix, and $\mathbf{d}=\left[\begin{array}{ll}u_{i} & v_{i}\end{array}\right]^{T}$ is vector of nodal displacements along $x$ and $y$ directions respectively. Superscript $T$ means transpose. The normal strain $\varepsilon_{x}$ and $\varepsilon_{y}$ along $x$ and $y$ directions respectively and the in-plane shear strain $\gamma_{x y}$ are: 
$\varepsilon_{x}=\frac{\partial u}{\partial x}, \varepsilon_{y}=\frac{\partial v}{\partial y}$ and $\gamma_{x y}=\frac{\partial u}{\partial y}+\frac{\partial v}{\partial x}$

Substitution of equations (14.b) and (15) into (16), gives:

$\boldsymbol{\varepsilon}=\left[\begin{array}{cc}\frac{\partial}{\partial x} & 0 \\ 0 & \frac{\partial}{\partial y} \\ \frac{\partial}{\partial y} & \frac{\partial}{\partial x}\end{array}\right]\left\{\begin{array}{l}u \\ v\end{array}\right\}=\left[\begin{array}{cc}\frac{\partial}{\partial x} & 0 \\ 0 & \frac{\partial}{\partial y} \\ \frac{\partial}{\partial y} & \frac{\partial}{\partial x}\end{array}\right] \mathbf{N d}=\mathbf{B d}$

Where $\boldsymbol{\varepsilon}=\left[\begin{array}{lll}\varepsilon_{x} & \varepsilon_{y} & \gamma_{x y}\end{array}\right]^{T}$ is the engineering strain vector, and $\mathbf{B}$ is the strain - displacement matrix defined as:
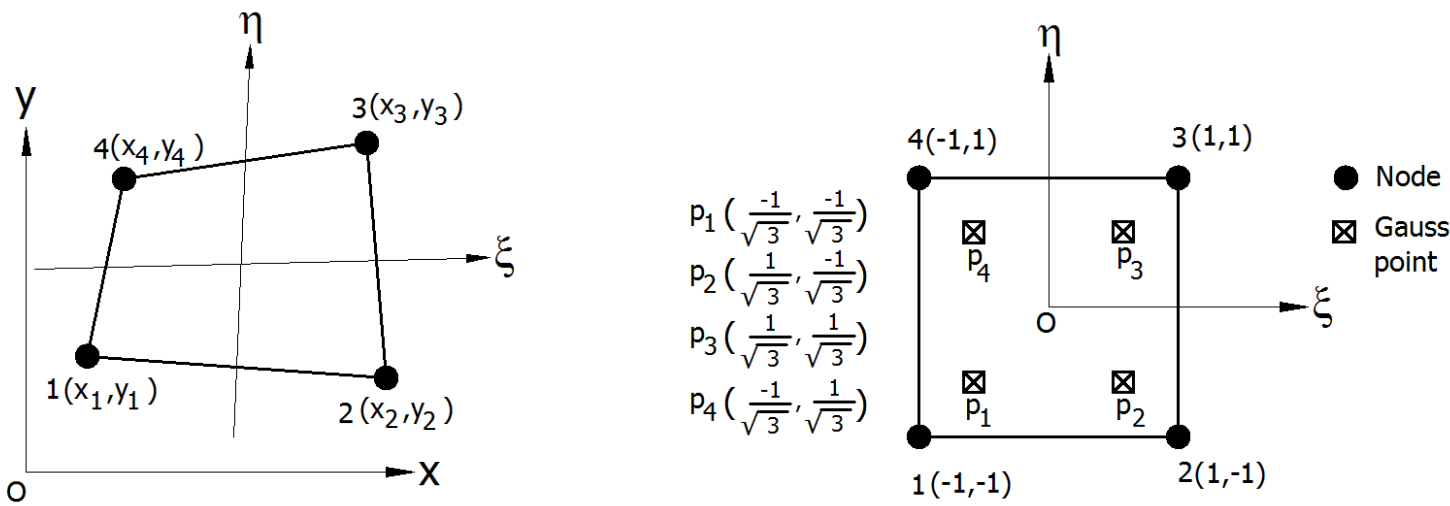

Fig. 4 Four Nodes Isoparametric Quadrilateral Element QUAD4 in (a) Cartesian Coordinates system and (b) Normalized Coordinates System.

$\mathbf{B}_{i}=\left[\begin{array}{cc}\frac{\partial N_{i}}{\partial x} & 0 \\ 0 & \frac{\partial N_{i}}{\partial y} \\ \frac{\partial N_{i}}{\partial y} & \frac{\partial N_{i}}{\partial x}\end{array}\right]=\frac{1}{|\jmath|}\left[\begin{array}{cc}\bar{b}_{i} & 0 \\ 0 & \bar{c}_{i} \\ \bar{c}_{i} & \bar{b}_{i}\end{array}\right]$

where $|\mathcal{J}|$ is the Jacobian defined as:

$|\mathcal{J}|=\left|\begin{array}{ll}\frac{\partial x}{\partial \xi} & \frac{\partial y}{\partial \xi} \\ \frac{\partial x}{\partial \eta} & \frac{\partial y}{\partial \eta}\end{array}\right|$

The coefficients $\bar{b}_{i}$ and $\bar{c}_{i}$ are:

$\bar{b}_{i}=\frac{\partial y}{\partial \eta} \frac{\partial N_{i}}{\partial \xi}-\frac{\partial y}{\partial \xi} \frac{\partial N_{i}}{\partial \eta}$ and $\bar{c}_{i}=\frac{\partial x}{\partial \xi} \frac{\partial N_{i}}{\partial \eta}-\frac{\partial x}{\partial \eta} \frac{\partial N_{i}}{\partial \xi}$

The normal components of stresses $\sigma_{x}$ and $\sigma_{y}$ along $x$ and $y$ directions respectively and the in-plane shear stress $\tau_{x y}$ are evaluated in case of plane strain condition as the following:

$\sigma=\mathbf{D} \varepsilon$ 
Where $\boldsymbol{\sigma}=\left[\begin{array}{lll}\sigma_{x} & \sigma_{y} & \tau_{x y}\end{array}\right]^{T}$ is the stress vector, and $\mathbf{D}$ is the elasticity matrix defined in plane strain condition as the following:

$[D]=\frac{E}{(1+v)(1-2 v)}\left[\begin{array}{ccc}1-v & v & 0 \\ v & 1-v & 0 \\ 0 & 0 & \frac{1-2 v}{2}\end{array}\right]$

Since, the computational model - in the present work - is linear and conservative (nondissipative). Therefore, the principal of virtual work can be applied to derive the governing equation of element's equilibrium in a matrix form; i.e.

$\delta U=\delta W$

Where $\delta U$ is the virtual change in the total strain energy and $\delta W$ is the virtual change in the work of the applied surface loads. In matrix form, equation (19.a) can be written as the following:

$\delta U=\int_{V} \delta \boldsymbol{\varepsilon}^{T} \boldsymbol{\sigma} d V=\delta \mathbf{d}^{T}\left(\int_{V} \mathbf{B}^{T} \mathbf{D} \mathbf{B} d V\right) \mathbf{d}=\delta \mathbf{d}^{T} \mathbf{K d}$

Where $V$ is the element volume; and $\mathbf{K}$ is the element stiffness matrix defined as:

$\mathbf{K}=\int_{V} \mathbf{B}^{T} \mathbf{D B} d V$

The work of external applied surface traction is:

$\delta W=\int_{S} \delta \mathbf{d}^{T}\left\{\begin{array}{c}0 \\ \sigma_{y}\end{array}\right\} d S=\delta \mathbf{d}^{T}\left(\int_{S} \mathbf{N}^{T}\left\{\begin{array}{c}0 \\ \sigma_{y}\end{array}\right\} d S\right)=\delta \mathbf{d}^{T} \mathbf{F}$

Where $S$ is the surface area upon which the traction $\left[\begin{array}{cc}0 & \sigma_{y}\end{array}\right]^{T}$ is applied; and $\boldsymbol{F}$ is the generalized element force vector defined as:

$\mathbf{F}=\int_{S} \mathbf{N}^{T}\left\{\begin{array}{c}0 \\ \sigma_{y}\end{array}\right\} d S$

Substituting by equations (19.b and d) into equation (19.a) and dividing by $\delta \mathbf{d}^{T} \neq 0$, we get:

$\mathbf{K d}=\mathbf{F}$

Equation (20) is the governing equation of equilibrium of the element in matrix form. Evaluation of element stiffness matrix equation (19.c) and element force vector equation (19.e) are performed numerically using $2 \times 2$ Gauss numerical integration rule. For example, consider the numerical integration of element stiffness matrix; equation (19.c) The first step is to map the integration onto the natural coordinates system; i.e.

$\mathbf{K}_{i j}=\int_{V} \mathbf{B}_{i}^{T} \mathbf{D B}_{j} d V=t \int_{-1}^{+1} \int_{-1}^{+1} \mathbf{B}_{i}^{T}(\xi, \eta) \mathbf{D B}_{j}(\xi, \eta)|\mathcal{J}(\xi, \eta)| d \xi d \eta$

Where $\mathcal{A}$ and $t$ are the element area and thickness respectively. The second step is to evaluate the last integrand in equation (20.a) at four Gauss points shown in Fig. 4(b) then multiply each term in a weighting factor $w_{p}=1$; as the following: 


$$
\mathbf{K}_{i j}=\sum_{p=1}^{p=4} \mathbf{B}_{i}^{T}\left(\xi_{p}, \eta_{p}\right) \mathbf{D B}_{j}\left(\xi_{p}, \eta_{p}\right)\left|\mathcal{J}\left(\xi_{p}, \eta_{p}\right)\right| w_{p}
$$

The same procedure is applied to integrate the element force vector; equation (19.e). However, the integration is performed on the top surface of the QUAD4 element. Thus the two - dimensional surface integration in equation (19.e) becomes one - dimensional line integration. The shape function of $\mathcal{N}_{i}$ isoparametric line element is:

$\mathcal{N}_{i}=\frac{1}{2}\left(1+\xi \xi_{i}\right)$

Two Gauss points are used to perform the integration numerically, their locations on the isoparametric line element are:

$\xi_{p 1}=-\frac{1}{\sqrt{3}}$, and $\xi_{p 1}=\frac{1}{\sqrt{3}}$

The Gauss - summation form of the element force vector is:

$\mathbf{F}=t \sum_{p=1}^{p=2} \mathcal{N}^{T}\left(\xi_{p}\right)\left\{\begin{array}{c}0 \\ \sigma_{y}\end{array}\right\} w_{p}$

\section{MESHING THE CACK TIP REGION AND REALIZATION OF THE $q$ FUNCTION}

Design of crack tip mesh characterizes the proposition of $q$ function. Figure 5 exemplifies a mesh of a crack tip region. Taking the crack tip as an origin of a local polar coordinate system, parameters controlling the mesh are: (1) crack mouth angle; $\varphi$, (2) number of elements in radial diction; NRING; (NRING $=3$ in Fig. 5), (3) number of elements in circumferential direction; NFAN; (NFAN $=6$ in Fig. 5), and (4) ratio between the outer radius of the rosette $R$ to half crack length $a$; this ratio is denoted by $\rho=\frac{R}{a}$. Elements in the first ring are always degenerated QUAD4. A degenerated QUAD4 element is obtained by assigning same coordinates to any adjacent nodes of the parent element. In the next section, the influences of aforementioned parameters on the accuracy of numerical results are investigated. 


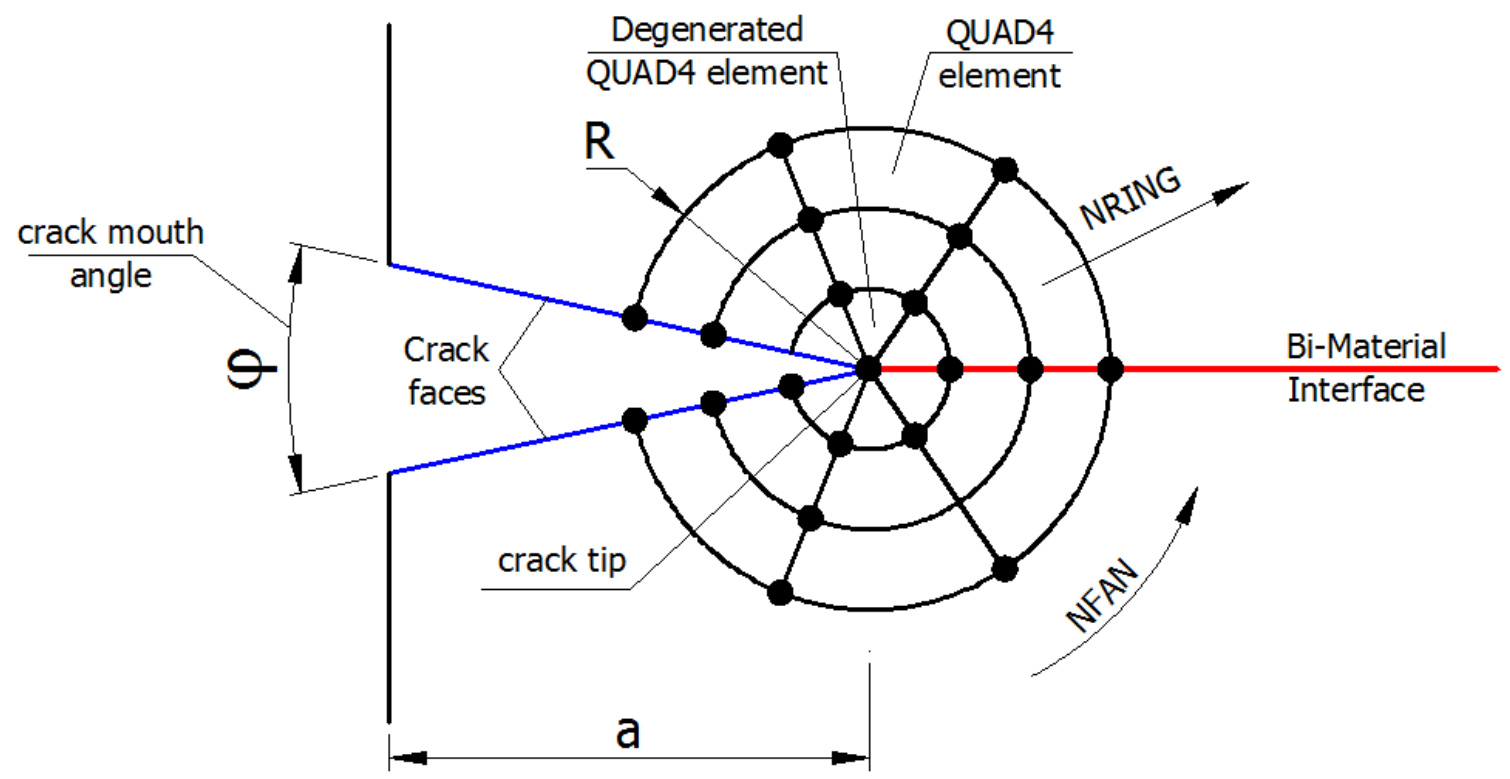

Fig. 5 Design Parameters Controlling Crack Tip Mesh

In this work, the domain of the equivalent energy integral, equation (13) is an annulus around the crack tip, Fig. 6 . The area of the domain is bounded by the two circular contours $\Gamma_{1}$ and $\Gamma_{4}$ and crack faces. Radii of the $\Gamma_{1}$ and $\Gamma_{4}$ are $R_{\Gamma_{1}}$ and $R_{\Gamma_{4}}$ respectively. The energy integral, equation (13), is calculated over the elements meshing the annulus $A$. The number of the elements is equal to NFAN. The number of annuli where, we can calculate the energy integral is equal to NRING. That means for each crack mesh we have NRING values of $J$ - integral. And according to the path - independent property of $J$ - integral, their values should be equal; equation (12.c). The values of are $R_{\Gamma_{1}}$ and $R_{\Gamma_{4}}$ are determined according to $\rho$ and NRING, where the radius $R$ is equally divided into NRING annuli.

To define the $\mathrm{q}$ function within a QUAD4 element, the first and second nodes of the element should be located on $\Gamma_{1}$ and third and fourth nodes on $\Gamma_{4}$ as shown in Fig. 6. Nodal values $Q_{i}$ of function $q$ are assigned to have the following values, Fig. 6:

$Q_{1}=Q_{2}=0$

$Q_{3}=Q_{4}=1$

The variation of $q$ within QUAD4 is expressed as:

$q(\xi, \eta)=N_{i}(\xi, \eta) Q_{i}$

Where $N_{i}$ is shape function of QUAD4 defined in equation (14.b). The spatial variation of $q$ has smooth conical form, Fig. 7(c). 


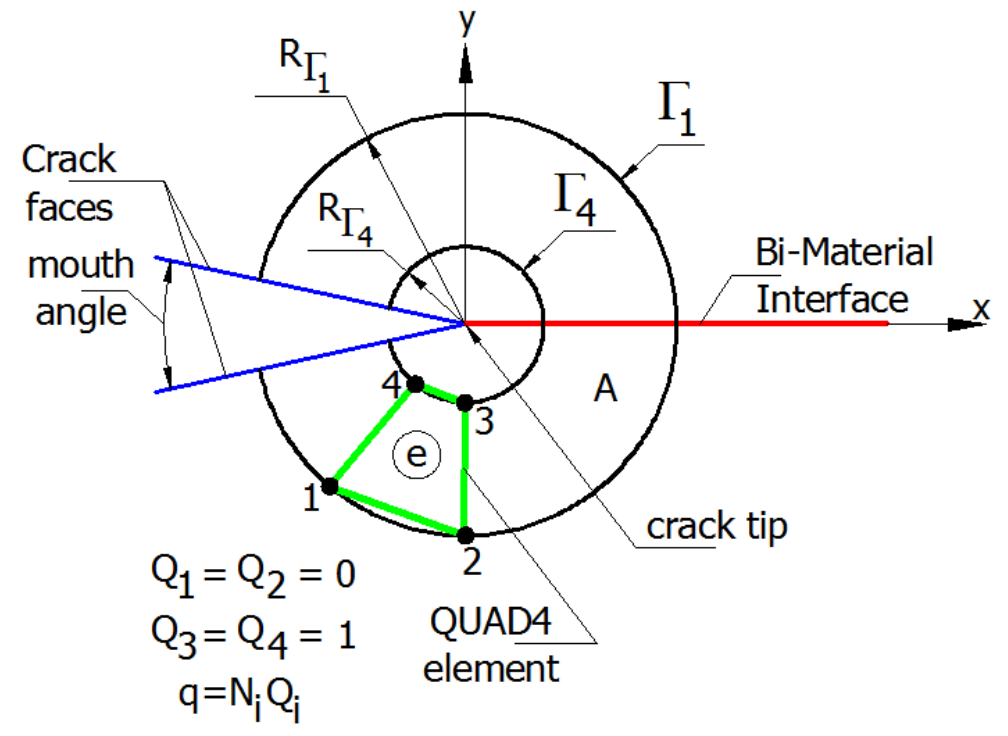

Fig. 6 Implementation of $q$ Function

It is worthy to mention that Shih et al [18] suggested two types of $q$; pyramid and plateau functions, Fig. 7(a) and (b). However, in this work, the conical form of $q$ is more convenient to fan-shaped rosette of elements around the crack tip, Fig. 5.

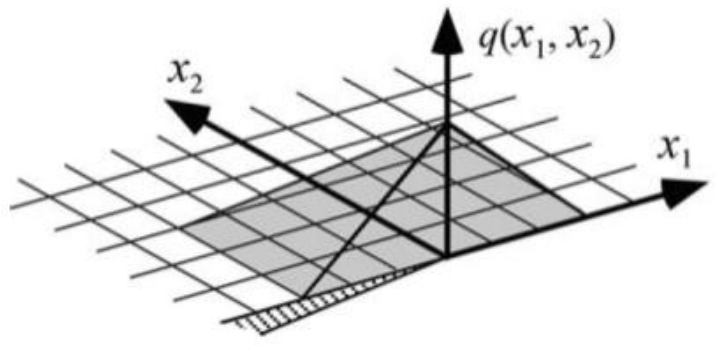

(a)

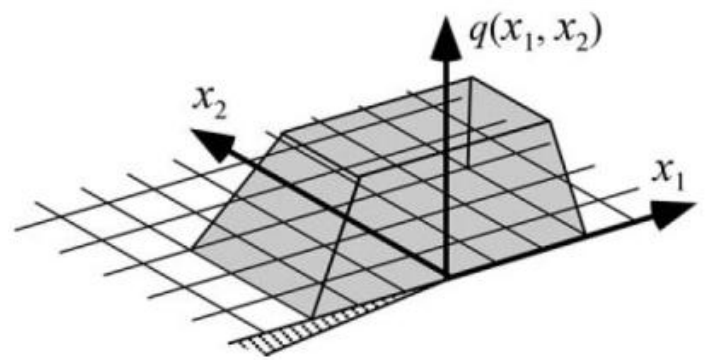

(b)

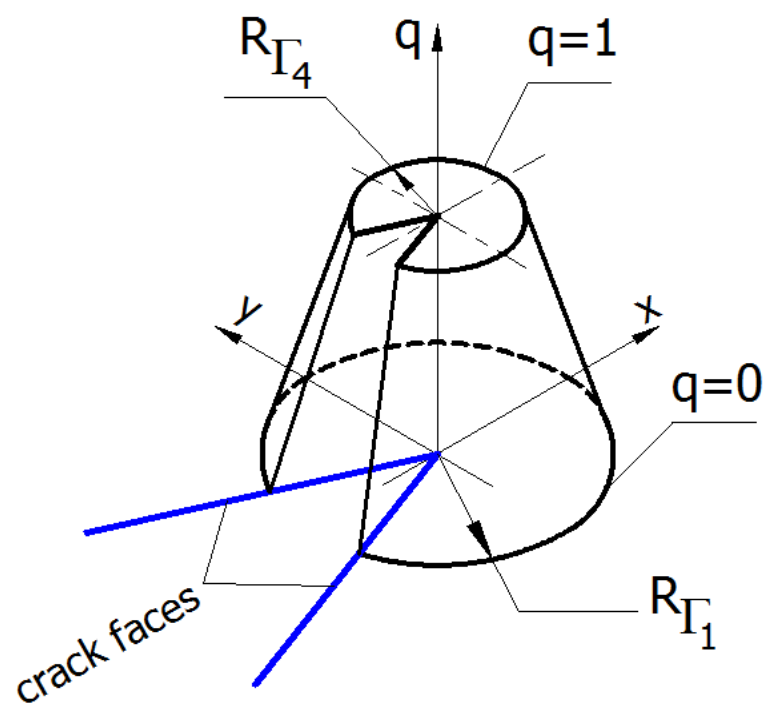

(c)

Fig. 7 Different Definitions of $q$ Function: (a) Pyramid and (b) Plateau as in Shih et al [20], (c) Conical as Proposed by in The Present Work.

The energy domain integral, equation (13) is calculated in the post processing stage of FEM computations, i.e. after the computations of displacement, strain and stress fields. The discretized form of the domain integral is written as the following:

$J=\sum_{n=1}^{N R I N G} \sum_{p=1}^{p=4} F\left(\xi_{p}, \eta_{p}\right)\left|\mathcal{J}\left(\xi_{p}, \eta_{p}\right)\right| w_{p}$

$F\left(\xi_{p}, \eta_{p}\right)=\left(\sigma_{x} \frac{\partial u}{\partial x}-w+\tau_{x y} \frac{\partial v}{\partial x}\right) \frac{\partial q}{\partial x}+\left(\tau_{y x} \frac{\partial u}{\partial x}+\sigma_{y} \frac{\partial v}{\partial x}\right) \frac{\partial q}{\partial y}$ 
$w=\frac{1}{2}\left(\sigma_{x} \varepsilon_{x}+\sigma_{y} \varepsilon_{y}+\tau_{x y} \gamma_{x y}\right)$

$\frac{\partial q}{\partial x_{i}}=\sum_{k=1}^{4} \sum_{m=1}^{2} \frac{\partial N_{k}}{\partial \xi_{m}} \frac{\partial \xi_{m}}{\partial x_{i}} Q_{k}$

$\frac{\partial u}{\partial x}=\frac{\partial N_{i}}{\partial x} u_{i}$ and $\frac{\partial v}{\partial x}=\frac{\partial N_{i}}{\partial x} v_{i}$

According to equation (11), numerical values of energy release rate $\mathbb{G}^{F E M}$ are calculated by equation (24.a); i.e.:

$\mathbb{G}^{F E M}=J$

In the present work, the effect of $\frac{a}{b}$ ratio is considered in numerical and analytical computations of stress intensity factor. Tada [21] expressed this effect as a factor $F\left(\frac{a}{b}\right)$ defined as:

$F\left(\frac{a}{b}\right)=\left\{1-0.025\left(\frac{a}{b}\right)^{2}+0.06\left(\frac{a}{b}\right)^{4}\right\} \sqrt{\sec \frac{\pi a}{2 b}}$

Thus, the numerical values of magnitude of stress intensity factor $\mathcal{K}^{F E M}$ are calculated as the following:

$\mathcal{K}^{F E M}=\cosh (\pi \epsilon) \sqrt{E^{*}} \sqrt{\mathbb{G}^{F E M}} F\left(\frac{a}{b}\right)$

The percentage errors in numerical results of the energy release rate $e_{\mathbb{G}}$ and the magnitude of stress intensity factors $e_{\mathcal{K}}$ are calculated as the following:

$e_{\mathbb{G}}=100 \times\left(\frac{\mathbb{G}^{F E M}-\mathbb{G}}{\mathbb{G}}\right)$

$e_{\mathcal{K}}=100 \times\left(\frac{\mathcal{K}^{F E M}-\mathcal{K}}{\mathcal{K}}\right)$

\section{NUMERICAL RESULTS: VALIDATION AND DISCUSSION}

The primary task in this section is the validation of the numerical computations of crack characteristics i.e. energy release rate and magnitudes of complex stress intensity factor. To achieve this goal, three crack problems are solved. The first is a thickness through crack in a single material system. The second problem is an interfacial crack in bi-material composite. The third one is the comparison of numerically calculated energy release rate with which experimentally measured in case of an interfacial crack in bi-material composite. Influences of varying parameters of crack tip mesh (i.e. $\varphi$, NRING, NFAN, and $\rho$ ) on crack characteristics are investigated as well.

\subsection{CRACK PROBLEM IN A SINGLE MATERIAL SYSTEM}

The mathematical model of this problem is shown in Fig. 3, by assigning the same mechanical properties to both layers; i.e:

$E_{1}=E_{2}=E_{c u}=120 \mathrm{GPa}$ and $v_{1}=v_{2}=v_{c u}=0.345$ 
Where $E_{c u}$ and $v_{c u}$ are Young's modulus and Poisson's ratio of the copper. The bi-material constant $\epsilon$, equation (2.a), is:

$\epsilon=0$

The mean Young's modulus of the single material system in plane strain condition; equation (7) is:

$E^{*}=136.21 \mathrm{GPa}$

Crack length and model dimensions are selected to be, Fig. 3, $2 a=20 \mathrm{~mm}, 2 b=140 \mathrm{~mm}$, and $h=70 \mathrm{~mm}$. Surface tensile stress (traction) is $\sigma_{y}=280 \mathrm{MPa}$. The analytical value of stress intensity factor, equation (5), considering the $\frac{a}{b}$ effect, equation (25.b), is:

$\mathcal{K}=K_{1}=K_{I}=\sigma_{y} \sqrt{\pi a} F\left(\frac{a}{b}\right)=50.238 \operatorname{MPa} \sqrt{m}$

The analytical value of the energy release rate, equation (8) is:

$\mathbb{G}=18.082 \mathrm{~kJ} / \mathrm{m}^{2}$

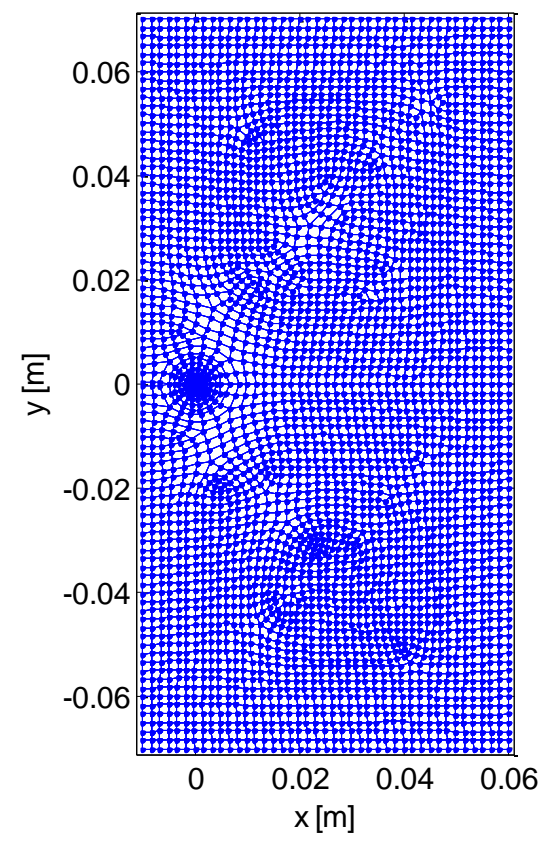

(a)

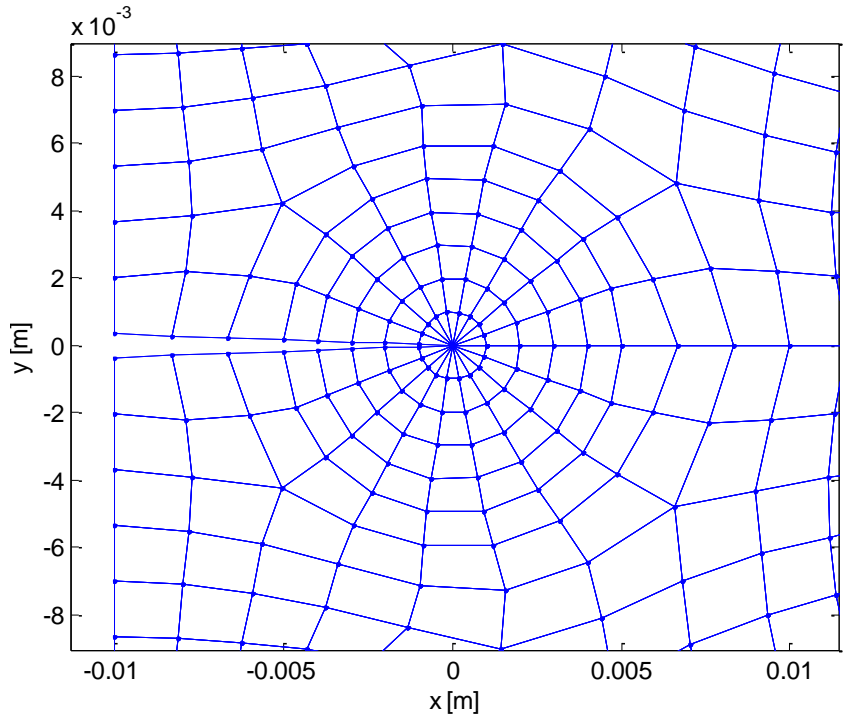

(b)

Fig. 8 (a) Finite Element Model of The Single Layer Through Thickness Crack Problem. (b) Crack Tip Mesh; $\varphi=4^{\circ}$, NRING $=5$, NFAN $=18, \rho=0.5$.

The finite element model is illustrated in Fig. 8(a) with an enlarged view of crack tip mesh in Fig. 8(b). The crack tip mesh parameters in Fig. 8(b) are: $\varphi=4^{\circ}$, NRING $=5$, NFAN $=18$, $\rho=0.5$. The total number of nodes and elements of the whole model are 3872 and 3747 
respectively. The numerical finite element results of stress intensity factor and energy release rate are listed in Table 1.1.

Table 1.1 FE Results of Crack Characteristics in The Single Layer System; Crack Mesh Parameters: $\varphi=4^{\circ}$, NRING $=5$, NFAN $=18, \rho=0.5$.

\begin{tabular}{c|c|c|c|c}
\hline \hline $\begin{array}{c}\text { Contour } \\
\text { number: }\end{array}$ & $\mathbb{G}^{F E M}, k J / m^{2}$ & $e_{\mathbb{G}}, \%$ & $\mathcal{K}^{F E M}, M P a \sqrt{m}$ & $e_{\mathcal{K}}, \%$ \\
\hline 1 & 16.451 & -9.023 & 47.337 & -5.775 \\
\hline 2 & 17.861 & -1.224 & 49.324 & -1.820 \\
\hline 3 & 18.221 & 0.767 & 49.819 & -0.836 \\
\hline 4 & 18.322 & 1.324 & 49.956 & -0.562 \\
\hline 5 & 18.373 & 1.608 & 50.026 & -0.422 \\
\hline \hline
\end{tabular}

Inspection of errors $e_{\mathbb{G}}$ and $e_{\mathcal{K}}$ reveals that the degenerated QUAD4 elements in the first contour result in high errors in $\mathbb{G}^{F E M}$ and $\mathcal{K}^{F E M}$ respectively. If the $J$ - integral contours is increased to NRING $=8$, the errors would be decreased, Table 1.2. However, the total number of nodes and elements of the whole mesh are changed to 3558 and 3439 respectively.

In order to study the influence of crack mesh parameters on crack characteristics, the number of $J$ contours is kept constant at NRING $=8$ and: (1) Number of elements in circumferential direction, NFAN is changed as shown in Fig. 9. (2) Ratio $\rho$ between domain radius $R$ and half crack length $a$ is varied as shown in Fig. 10; (3) Amount of crack mouth angle $\varphi$ is altered as depicted in Fig. 11.

Table 1.2 FE Results of Crack Characteristics in The Single Layer System; Crack Mesh Parameters: $\varphi=4^{\circ}$, NRING $=8, \mathrm{NFAN}=18, \rho=0.5$.

\begin{tabular}{c|c|c|c|c}
\hline \hline $\begin{array}{c}\text { Contour } \\
\text { number: }\end{array}$ & $\mathbb{G}^{F E M}, \mathrm{~kJ} / \mathrm{m}^{2}$ & $e_{\mathbb{G}}, \%$ & $\mathcal{K}^{F E M}, M P a \sqrt{m}$ & $e_{\mathcal{K}}, \%$ \\
\hline 1 & 16.612 & -8.132 & 47.568 & -5.315 \\
\hline 2 & 18.000 & -0.454 & 49.516 & -1.438 \\
\hline 3 & 18.350 & 1.481 & 49.995 & -0.485 \\
\hline 4 & 18.444 & 2.003 & 50.123 & -0.229 \\
\hline 5 & 18.490 & 2.254 & 50.185 & -0.106 \\
\hline 6 & 18.519 & 2.419 & 50.225 & -0.026 \\
\hline 7 & 18.542 & 2.545 & 50.256 & 0.036 \\
\hline 8 & 18.561 & 2.651 & 50.282 & 0.087 \\
\hline \hline
\end{tabular}

Analyses of figures 9 to 11 lead to the following two important conclusions: (a) Results related to the first contour are a little bit away from the analytical ones, (b) Varying of crack mesh parameters has minor influence on the results of crack characteristics at least on the engineering level. On the light of both conclusions, we will solve the next two crack problems, subsections 5.2 and 5.3. 


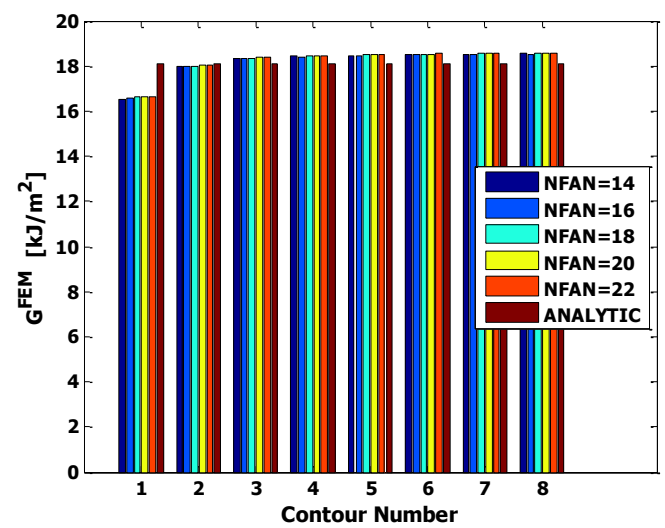

(a)

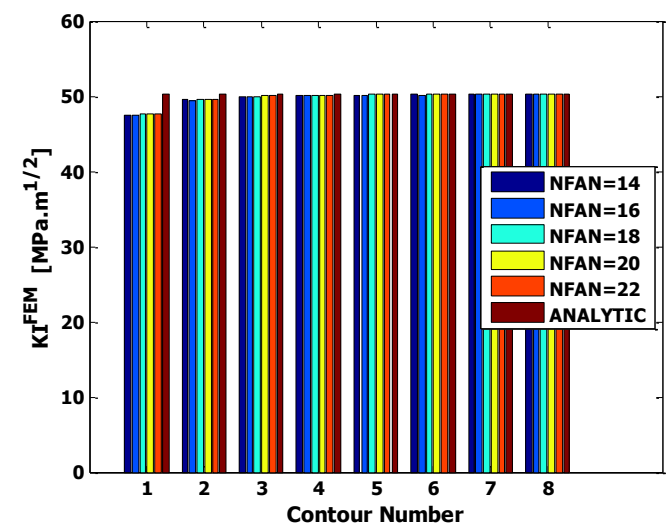

(b)

Fig. 9 Effect of varying NFAN on (a) Energy release rate; $\mathbb{G}^{F E M}$ and (b) Stress intensity factor; $\mathcal{K}^{F E M}$, at $\varphi=4^{\circ}, \mathrm{NRING}=8$, and $\rho=0.5$.
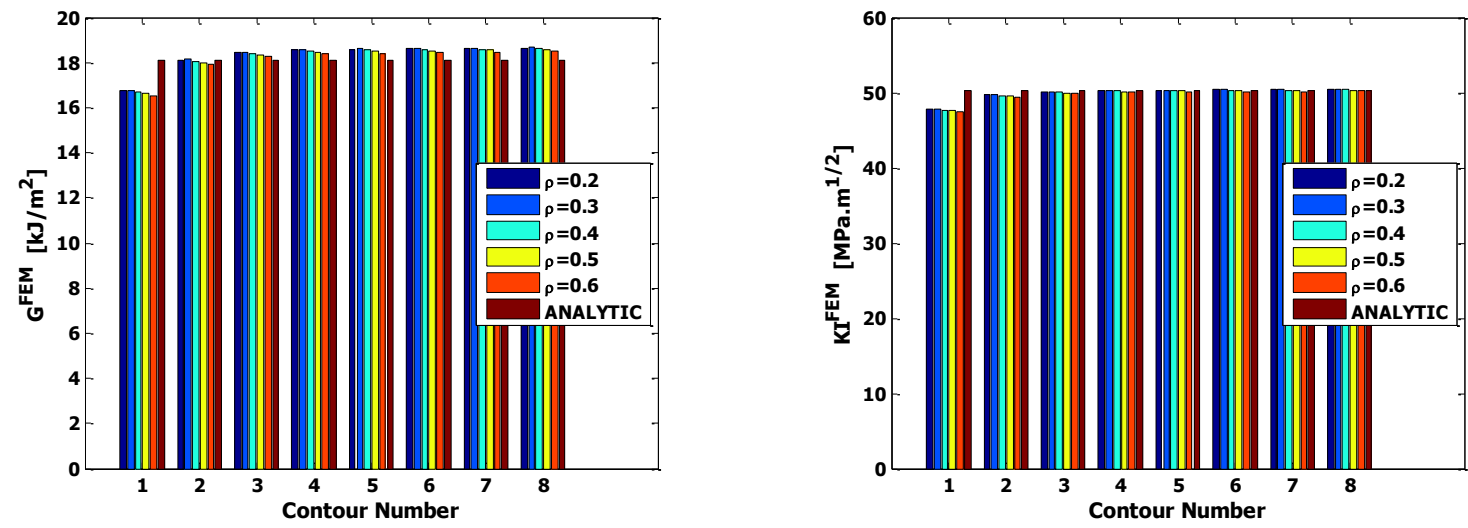

Fig. 10 Effect of varying $\rho$ on (a) Energy release rate; $\mathbb{G}^{F E M}$ and (b) Stress intensity factor; $\mathcal{K}^{F E M}$, at $\varphi=4^{\circ}, \mathrm{NRING}=8$, and NFAN $=18$.
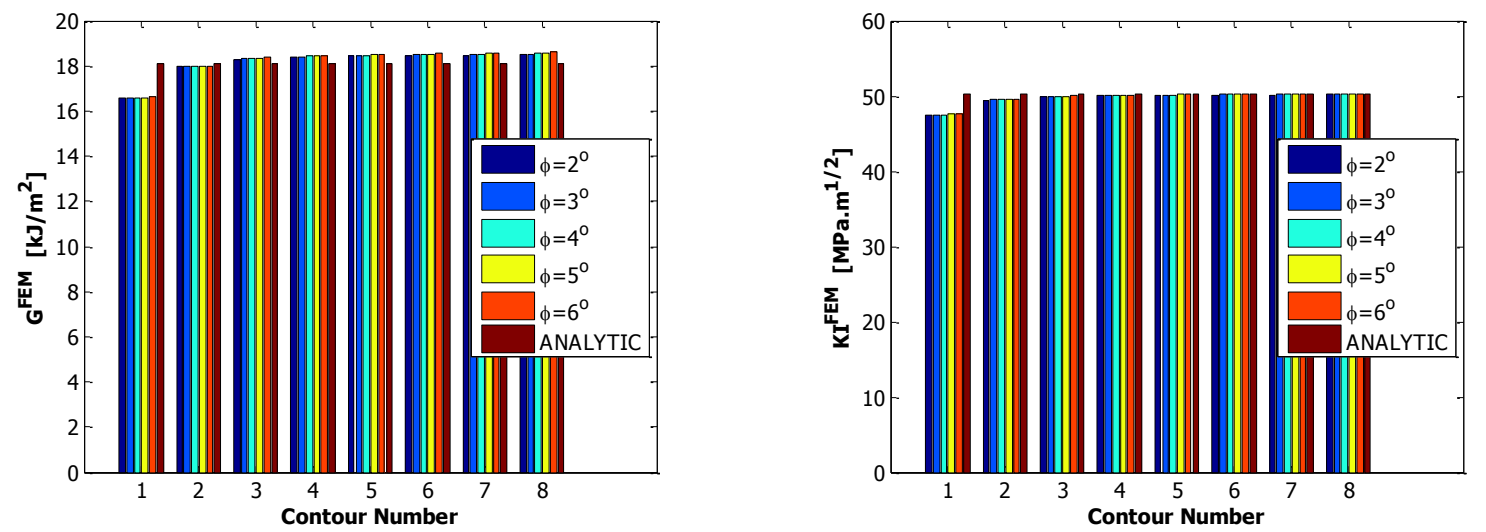

Fig. 11 Effect of varying $\varphi$ on (a) Energy release rate; $\mathbb{G}^{F E M}$ and (b) Stress intensity factor; $\mathcal{K}^{F E M}$; at $\rho=0.5, \mathrm{NRING}=8$, and NFAN $=18$. 


\subsection{INTERFACIAL CRACK PROBLEM IN BI-MATERIAL COMPOSITE}

The second crack problem is the interfacial thickness through crack in a bi-material composite. The mathematical model of the problem is illustrated in Fig. 3. Material properties are selected to be $E_{1}=E_{A l 2 O 3}=432.6 \mathrm{GPa}, E_{2}=E_{c u}=120 \mathrm{GPa}, v_{1}=v_{A l_{2} O_{3}}=0.207$ and $v_{2}=v_{c u}=0.345$. Where $E_{A l 2 O 3}$ and $v_{\mathrm{Al}_{2} \mathrm{O}_{3}}$ are Young's modulus and Poisson's ratio of aluminum oxide respectively. Model dimensions and loads are the same as in the first problem; i.e. $a=20 \mathrm{~mm}, 2 b=140 \mathrm{~mm}, h=70 \mathrm{~mm}$, and $\sigma_{y}=280 \mathrm{MPa}$. The analytical value of complex stress intensity factor, equation (3.a), is:

$K=(49.505-9.0932 i) M P a \sqrt{m}$

The magnitude of complex stress intensity factor considering the $\frac{a}{b}$ effect, equations (3.b) and (25.b), is:

$\mathcal{K}=50.333 \mathrm{MPa} \sqrt{m}$

The analytical value of the energy release rate, equation (8) is:

$\mathbb{G}=11.701 \mathrm{~kJ} / \mathrm{m}^{2}$

The deformed finite element model is illustrated in Fig. 12(a) with an enlarged view of deformed crack tip mesh in Fig. 12(b). The crack mesh parameters in Fig. 12(b) are: $\varphi=4^{\circ}$, NRING $=8$, NFAN $=18, \rho=0.25$. The total number of nodes and elements are 3475 and 3353 respectively. The finite element results of the magnitude of complex stress intensity factor and the energy release rate are listed in Table 2.

Disregarding the computations corresponding to the first ring (contour), the maximum error in $\mathbb{G}^{F E M}$ and $\mathcal{K}^{F E M}$ are $1.608 \%$ and $1.707 \%$ respectively. The minimum errors in are $\mathbb{G}^{F E M}$ and $\mathcal{K}^{F E M}$ are $-0.996 \%$ and $-0.422 \%$ respectively, Table 2 . 


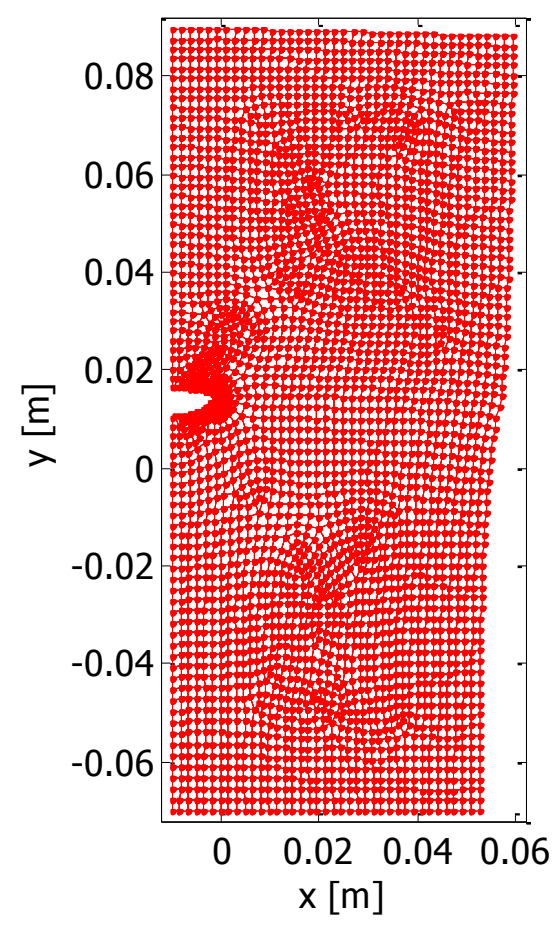

(a)

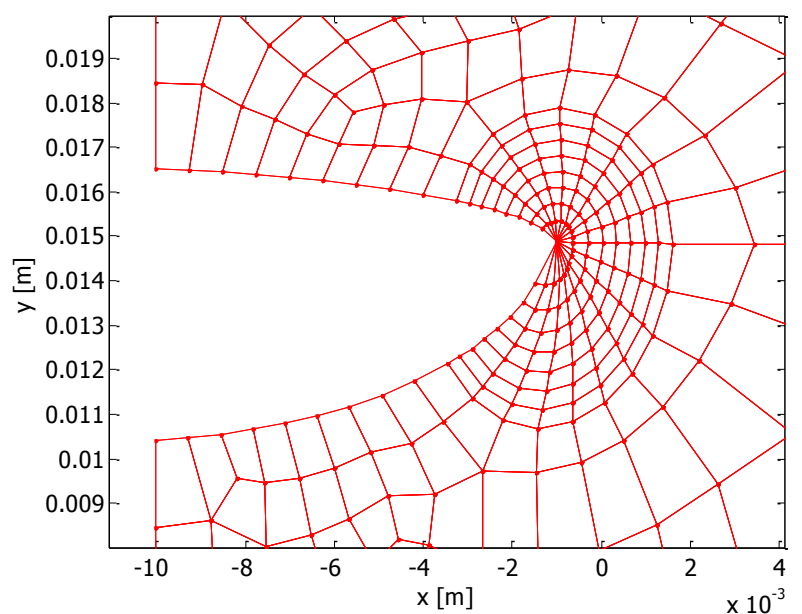

(b)

Fig. 12 (a) Deformed FE Model of The Interfacial Crack Problem in $\mathrm{Al}_{2} \mathrm{O}_{3} / \mathrm{Cu}$

Composite. (b) Magnified View of Deformed Crack Tip Mesh. Crack Mesh Parameters: $\varphi=4^{\circ}, \mathrm{NRING}=8, \mathrm{NFAN}=18, \rho=0.25$. All deformations are multiplied by 100 for illustrative purposes.

Table $2 \mathrm{FE}$ Results of Interfacial Crack Characteristics in $\mathrm{Al}_{2} \mathrm{O}_{3} / \mathrm{Cu}$ Composite; Crack Mesh Parameters: $\varphi=4^{\circ}, \mathrm{NRING}=8$, NFAN $=18, \rho=0.25$.

\begin{tabular}{c|c|c|c|c}
\hline \hline $\begin{array}{c}\text { Contour } \\
\text { number: }\end{array}$ & $\mathbb{G}^{F E M}, k J / m^{2}$ & $e_{\mathbb{G}}, \%$ & $\mathcal{K}^{F E M}, M P a \sqrt{m}$ & $e_{\mathcal{K}}, \%$ \\
\hline 1 & 10.722 & -8.368 & 47.597 & -5.437 \\
\hline 2 & 11.584 & -0.996 & 49.474 & -1.707 \\
\hline 3 & 11.791 & 0.774 & 49.915 & -0.832 \\
\hline 4 & 11.840 & 1.193 & 50.018 & -0.626 \\
\hline 5 & 11.862 & 1.374 & 50.063 & -0.537 \\
\hline 6 & 11.874 & 1.479 & 50.089 & -0.486 \\
\hline 7 & 11.882 & 1.552 & 50.107 & -0.450 \\
\hline 8 & 11.889 & 1.608 & 50.121 & -0.422 \\
\hline \hline
\end{tabular}

\subsection{COMPARISION WITH EXPERIMENTAL RESULTS}

Sun et al [22] measured experimentally the fracture toughness of delamination between plasma-enhanced chemical vapor deposition SiNx islands (hard coating) and a polyimide (PI) substrate (soft substrate), Fig. 13. Such structure is frequently found in flexible electronics industry. They applied tensile strain and measured the deboned length $(2 a=L-d)$, Fig. 13(b); where $d$ is the remaining bonded length of the coating (island), Fig. 13(a). After rigorous theoretical and numerical analyses, they related the applied strain with the fracture energy, Fig. 13(c). They proposed a symmetric mathematical model for their problem, Fig. 13(d) and assumed plane strain state of deformation. Mechanical properties of both coating and substrate materials are listed in Table 3.1. 
Table 3.1 Dimensions, Loading and Mechanical Properties for Symmetric Sun et al [22]

Model corresponding to $h=1 \mu \mathrm{m}, L=20 \mu \mathrm{m}$ and $\varepsilon_{a p p}=0.028$

\begin{tabular}{l|c}
\hline \hline Dimensions: & $\frac{\text { Mechanical properties: }}{\text { Coating: }}$ \\
& $E_{\mathrm{SiNx}}=200 \mathrm{GPa}$ \\
Coating length $L=20 \mu \mathrm{m}$. & $v_{\mathrm{SiNx}}=0.3$ \\
Coating thickness $h=1 \mu \mathrm{m}$. & \\
& Substrate: \\
Substrate length $S=30 \mu \mathrm{m}$. & $E_{\mathrm{PI}}=9.2 \mathrm{GPa}$ \\
Substrate thickness $H=12.5 \mu \mathrm{m}$. & $v_{\mathrm{PI}}=0.3$ \\
\hline \hline
\end{tabular}

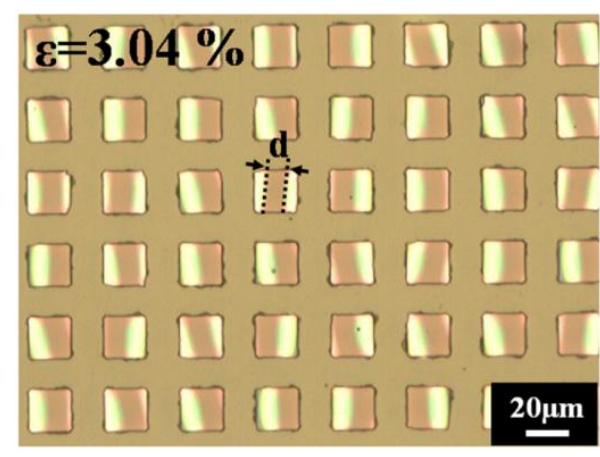

(a)

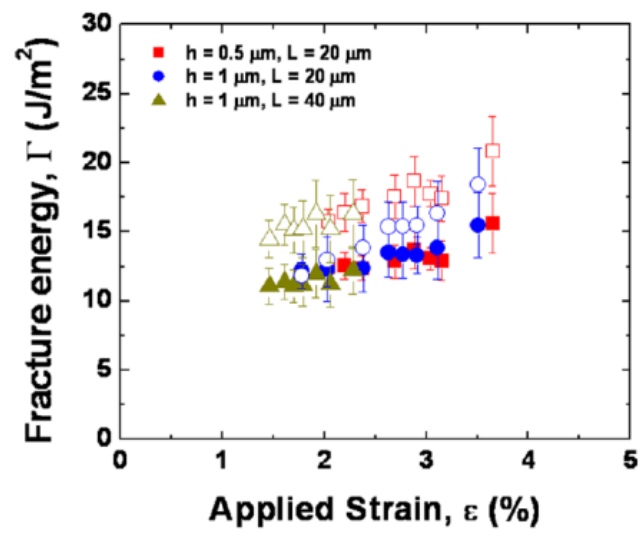

(c)

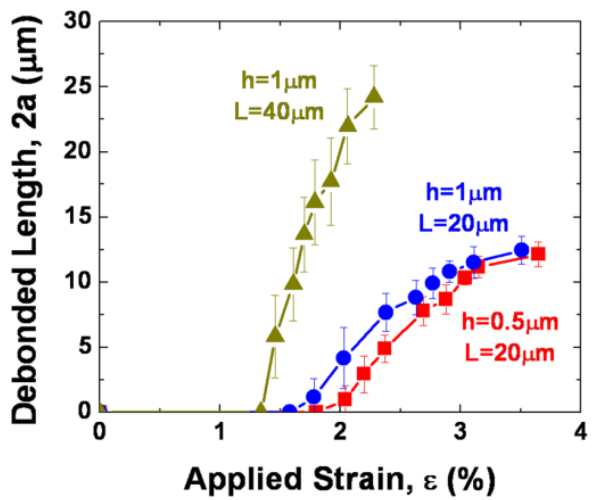

(b)

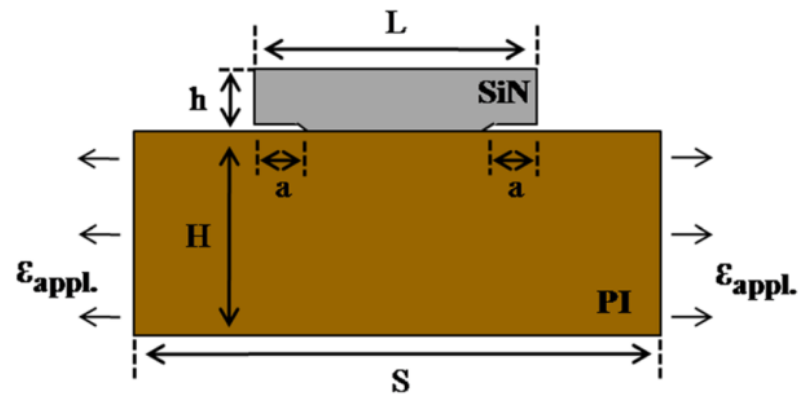

(d)

Fig. 13 (a) Sun et al Experiment, [22] (b) Relation between Applied Strain and Delaminated Interfacial Crack Length, [22] (c) Relation Between Applied Strain and Fracture Energy of Delamination, [22]. (d) Sun et al Symmetric Model. [22].

In this section, we solve for interfacial debonding energy corresponding to applied strain $\varepsilon_{a p p}=0.028$ and coating thickness $h=1 \mu \mathrm{m}$ and coating length $L=20 \mu \mathrm{m}$. From Fig. 13(b), we read the length of delaminated crack as $2 a=8 \mu \mathrm{m}$. The corresponding fracture energy is taken from Fig. 13(c) as $\mathbb{G}=13 \mathrm{~J} / \mathrm{m}^{2}$. The proposed finite element model in the present study is shown in Fig. 14(a). Mesh parameters of crack tip regions, Fig. 14(b), are: $\varphi=2^{\circ}, \quad$ NRING $=8, \quad N F A N=18$, and $\rho=0.15$. Boundary conditions are: (1) Horizontal displacement of the left edge nodes is fixed. (2) Vertical displacement of the bottom edge nodes is fixed. The applied strain is realized as applied horizontal displacement $\hat{u}=0.42 \mu \mathrm{m}$ to the nodes of right substrate edge. The deformed model is shown in Fig. 14(c) and (d). Contour results of energy release rate $\mathbb{G}^{F E M}$ (delamination fracture energy) and the 
corresponding errors are listed in Table 3.2. Excluding the result of the first contour, maximum and minimum errors are $-3.342 \%$ and $-0.085 \%$ corresponding to the second and fifth contour respectively.

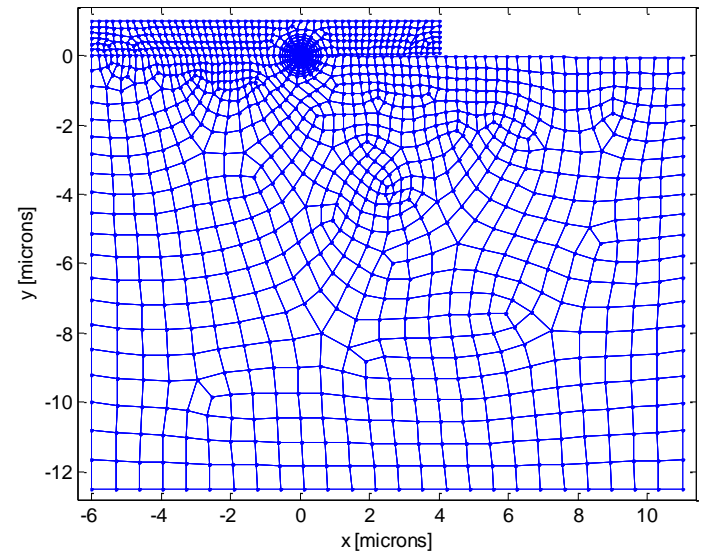

(a)

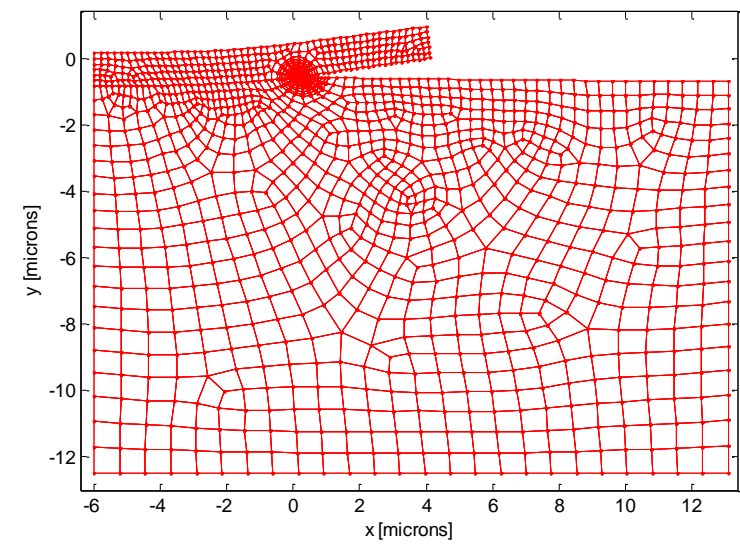

(c)

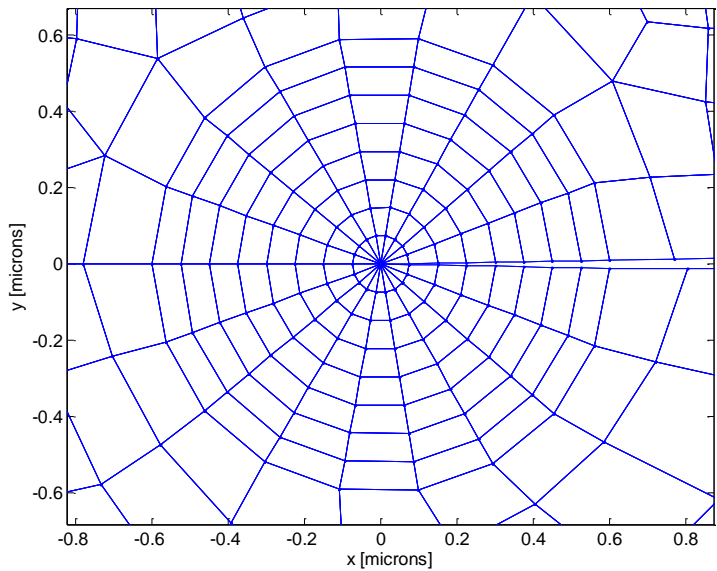

(b)

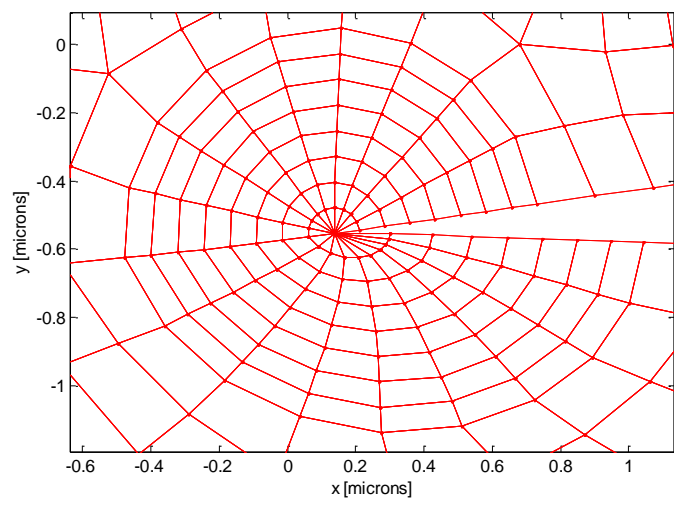

(d)

Fig. 14 (a) Present Work FE Model. (b) Enlaged View of Interfacial Crack.

Crack Mesh Parameters are: $\varphi=2^{\circ}$, NRING $=8$, NFAN $=18$, and $\rho=0.15$. (c)

Deformed FE model. (d) Magnified View of Deformed Crack Tip Mesh. Deformations are multiplied by 4 for Illustrative Purposes

Table 3 Present Work Computations of Delamination Fracture Toughness between SiNx and PI corresponding to $h=1 \mu \mathrm{m}, L=20 \mu \mathrm{m}$ and $\varepsilon_{a p p}=0.028$

\begin{tabular}{c|c|c}
\hline $\begin{array}{c}\text { Contour } \\
\text { number: }\end{array}$ & $\mathbb{G}^{F E M}, \mathrm{~J} / \mathrm{m}^{2}$ & $e_{\mathbb{G}}, \%$ \\
\hline 1 & 11.628 & -10.551 \\
\hline 2 & 12.566 & -3.342 \\
\hline 3 & 12.844 & -1.201 \\
\hline 4 & 12.933 & -0.516 \\
\hline 5 & 12.989 & -0.085 \\
\hline 6 & 13.031 & 0.236 \\
\hline 7 & 13.065 & 0.499 \\
\hline 8 & 13.095 & 0.728 \\
\hline
\end{tabular}




\section{CONCLUSION}

This paper discussed the finite element computations of fracture characteristics of interfacial crack in isotropic, linear and elastic bi-material composite. It presented: (1) Conical form of the assumed crack extension vector; $q$ function and (2) Four parameters controlling the design of crack tip mesh with non-singular 4 nodes isoparametric quadrilateral elements. Elastic fracture characteristics are computed in the post processing stage using the counter $J$ - integral via equivalent energy domain integral. Results of the present work, showed good agreement with both referenced and experimental values.

\section{References}

[1] Rice J.R. and Sih G.C., "Plane Problems of Cracks in Dissimilar Materials", Journal of Applied Mechanics, 32, 1965, pp. 418-423.

[2] Qian G., Nakamura and Bemdt C.C, "Effects of Thermal Gradient and Residual Stresses on Thermal Barrier Fracture", Mechanics of Materials, 27, 1998, pp. 91-110.

[3] Joshua L. Strom and John P. Parmigiani, "Transition of Crack Path at Bi-Material Interfaces”, Engineering Fracture Mechanics, 115, 2014, pp. 13-21.

[4] Zhigang Suo, "Singularities Interacting with Interfaces and Cracks", Int. J. Solids Structures, Vol. 25, No. 10, 1989, pp. 1133 - 1142.

[5] Chow W.T. and Atluri S.N., "Finite Element Calculation of Stress Intensitu Factors for Interfacial Crack Using Virtual Crack Closure Integral”, Computational Mechanics, 16, 1995, pp. $417-425$.

[6] Rice J.R., "Elastic Fracture Mechanics Concepts for Interfacial Cracks", Journal of Applied Mechanics, 55, 1988, pp. 98-103.

[7] Hutchinson J.W. and Suo Z., "Mixed Mode Cracking in Layered Materials", 29, Advances in Applied Mechanics, 1992, pp. 63-191.

[8] Hutchinson J.W., Mear M.E. and Rice J.R., "Crack Paralleling an Interface Between Dissimilar Materials”, Journal of Applied Mechanics, 54, 1987, pp. 828-832.

[9] Anderson, T. L. Fracture Mechanics - Fundamentals and Applications. 3nd ed., Boca Raton: CRC, 2005.

[10] Meinhard Kuna, Finite Elements in Fracture Mechanics, Springer, 2013.

[11] Sukumar N., Chopp D.L., Moes N. and Belytschko T., "Modeling Holes and Inclusions by Level Sets in The Extended Finite Element Method", Comput. Mthods Appl. Mech. Engrg., 190, 2001, pp. 6183-6200.

[12] Sukumar N. and Prevost J.H., "Modeling quasi-static crack growth with the extended finite element method Part I: Computer implementation", International Journal of Solids and Structures, 40, 2003, pp. 7513-7537.

[13] Goangseup Zi and Belytschko Ted, "New Crack-Tip Elements for XFEM and Applications to Cohesive Cracks", Int. J. Numer. Meth. Engng., 57, 2003; pp. 2221-2240.

[14] Sukumar N., Huang Z.Y., Prevost J.H. and Suo Z., "Partition of Unity Enrichment for Bi-Material Interface Cracks”, Int. J. Numer. Meth. Engng, 59, 2004, pp. 1075-1102.

[15] http://www.automesh2d.com/default.htm.

[16] Rice, J. R. "A Path Independent Integral and the Approximate Analysis of Strain Concentration by Notched and Cracks." Journal of Applied Mathematics. 35 (1968): 379-386. 
[17] Nikishkov G.P. and Atluri S.N., "Calculation of Fracture Mechanics Parameters for an Arbitrary Three-Dimensional Crack, by The Equivalent Domain Integral Method”, Int. J. Numer. Meth. Engng, 24, 1987, pp. 1801-1821.

[18] Gu P., Dao M., and Asaro R.J., "A Simplified Method for Calculating the Crack-Tip Field of Functionally Graded Materials Using The Domain Integral", Journal of Applied Mechanics, 66, 1999, pp. 101 - 108.

[19] E. Onate, Structural Analysis with the Finite Element Method. Linear Statics. Volume 1. Basis and Solids, Springer, 2009.

[20] Shih, C.F., Moran, B., and Nakamura T., "Energy Release Rate Along a ThreeDimensional Crack Front in a Thermally Stressed Body." International Journal of Fracture, Vol. 30, 1986, pp. 79-102.

[21] Tada H., Paris P.C., and Irwin G.R., The Stress Analysis of Cracks Handbook, $3^{\text {rd }}$ ed., ASME Press, New York, 2000.

[22] Jeong-Yun Sun, Nanshu Lu, Kyu-Hwan Oh, Zhigang Suo, and Joost J. Vlassak, "Islands Stretch Test for Measuring the Interfacial Fracture Energy Between a Hard Film and a Soft Substrate", Journal of Applied Physics 113, no. 22: 223702, 2013. Available online at http://nrs.harvard.edu/urn-3:HUL.InstRepos:12375010. 